\title{
Evaluation of a Nanostructured Microwave Absorbent Coating Applied to a Glass Fiber/Polyphenylene Sulfide Laminated Composite
}

\author{
L. C. Folgueras ${ }^{1,2 *}$, M. A. Alves ${ }^{1}$, M. C. Rezende ${ }^{1}$ \\ ${ }^{1}$ Divisão de Materiais, Instituto de Aeronáutica e Espaço - IAE, \\ Praça Mal. Eduardo Gomes, 50, CEP 12228-904, São José dos Campos, SP, Brasil \\ ${ }^{2}$ Universidade de Taubaté, Departamento de Engenharia Mecânica, \\ Rua Daniel Danelli s/n (Campus da Juta), CEP 12060-440, Taubaté, SP, Brasil.
}

Received: June 27, 2013; Revised: January 12, 2014

\begin{abstract}
The rapid coating of composites with absorbing paints yields materials that can absorb microwave radiation and still have approximately the same specific mass of the original composites. The use of paints with specific electromagnetic properties together with carbon- nanotube-based materials allows the production of structural materials of interest to the aeronautical industry. Thus, the objective of this study was to produce and characterize the electromagnetic properties of a material consisting of a microwave absorbing coating (carbon nanotubes and polyurethane) applied to a laminated composite made with polyphenylene sulfide and glass fiber. The attenuation of microwaves (8 to $12 \mathrm{GHz}$ ) incident on this material was evaluated using the waveguide technique to determine whether this material can be used as an absorbing structural material. The results show that the material absorbs up to $90 \%$ of the energy of the incident microwave, indicating that the material is an efficient absorber of microwave radiation.
\end{abstract}

Keywords: radar absorbing material, radar absorbing structures, nanocomposites

\section{Introduction}

Microwave absorbing material, also know as radar absorbing material or RAM, is a class of materials that attenuates the reflection of electromagnetic radiation, especially in the microwave frequency range. RAMs are produced in many forms (e.g., paints, composites, sheets, and thin films) by dispersing absorbing centers, which interact with the incident electromagnetic radiation, in different types of polymer matrices. RAMs can be applied to load-bearing structures or materials to create radar absorbing structures (RAS). Microwave absorbers have been used to prevent or minimize reflections from aircraft, ships and other military vehicles, large structures, and to cover the walls of anechoic chambers.

The application of paint to a substrate can change the surface impedance and impedance matching in such a way that the painted substrate attenuates the reflection of microwaves. The absorbing properties of a painted substrate are related to the proper impedance matching between the painted surface and air. The combination of different materials results in different impedance values and, consequently, different absorbing characteristics ${ }^{1}$.

One the goals of structural materials technology is to develop new materials, combining properties such as high mechanical resistance and rigidity, and low specific mass with the purpose to produce, for instance, structural composites for aerospace applications. The combination of structural composite materials with RAMs allows the production of functional materials, which can be also used in microwave applications s.3. $^{2,3}$.

Several types of polymer matrices can be used in the production of nanostructured absorbing materials ${ }^{4}$; but the choice of a matrix depends on the type of application.

*e-mail: luiza@ita.br
Polyurethane and thermoset resins such as epoxy, and thermoplastic polymers such as polyphenylene sulfide (PPS) have been used in the aeronautical industry because of their mechanical strength, erosion resistance and ability to withstand physical and chemical extremes ${ }^{5}$.

Carbon nanotubes (CNTs) is another class of materials with interesting and useful properties. The excellent thermal and electrical conductivities and the small dimensions of CNTs have motivated the development of new materials, including conductive plastics ${ }^{6,7}$. The use of paints with specific electromagnetic properties in conjunction with fibrous materials such as CNTs enables the production of structural materials that can absorb electromagnetic radiation. One objective of the present work was to evaluate the electromagnetic properties of a microwave absorbing coating produced with CNTs dispersed in a polyurethane matrix and applied to the surface of a glass fiber/polyphenylene sulfide (GF/PPS) laminated composite. Another objective was to evaluate the absorbent paint to determine whether it can be used with other composite materials such as those made with glass or carbon fibers and different types of resins.

Other composite-based RAMs have already been produced in our laboratory; the innovation aspect of the present study is in the use of a composite made with GF/ $\mathrm{PPS}^{2,3,8}$. Because of its mechanical, physical and chemical properties, this thermoplastic has been gaining popularity in the aeronautical industry, replacing other conventional materials in many applications. Moreover, the production of RAMs using PPS thermoplastic matrices reduces costs and this polymer has the additional advantage of being fire resistant, meeting the safety requirements of the aeronautical industry. 


\subsection{Propagation of electromagnetic wave in the interior of a material}

The amplitude of an electromagnetic wave propagating through a conducting material decreases as the wave moves

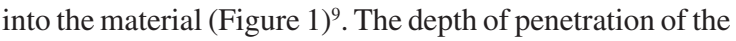
wave and the attenuation of the electric field at the surface of a material are parameters relevant to the design of thin microwave absorbing coatings ${ }^{10}$. In a conducting medium (e.g. metals), the depth of penetration of the electromagnetic wave is inversely proportional to the electrical conductivity of the medium, and most of the energy of an incident wave is reflected by the surface of conducting materials ${ }^{9,11}$.

Materials with high conductivity and low capacity to store energy, such as metals, have high dielectric losses. In this situation, the depth of penetration of the electromagnetic wave in these materials is minimal and they behave as reflectors (high reflectivity, attenuation of the incident electromagnetic wave is close to $0 \%$ ). In materials with low dielectric losses, the depth of penetration of the electromagnetic wave is larger. As a result, little energy is absorbed by the material and it is transparent to electromagnetic radiation. In the case of absorbent materials, most of the incident electromagnetic energy is absorbed. For this to happen, there must be a balance between the values of the electric conductivity, electric loss factor and the depth of penetration of the electromagnetic wave, which must be smaller than the total thickness of the material ${ }^{1,12,13}$.

The propagation of electromagnetic waves in a layered material is a complex process; it depends on the impedance values at the layer interfaces, which, in turn, affect the transmission and reflection of waves at these interfaces ${ }^{14}$. In layered materials, each layer behaves in a particular way, absorbing or reflecting the incident wave. This phenomenon has been studied using computer simulations involving complex algorithms ${ }^{11}$. For instance, if the concentration of absorbing centers in a given layer is high, the conductivity of this layer is probably high, and the layer may reflect electromagnetic waves. On the other hand, low concentration of absorbing centers favors the absorption of electromagnetic energy within the layer. The perfect impedance matching of materials with similar electrical properties allows the wave to first penetrate the layered material and then to be absorbed. Thus, the combination of different materials in layers with different thicknesses results in materials that behave as absorbers or reflectors of electromagnetic energy depending on how these layers are arranged.

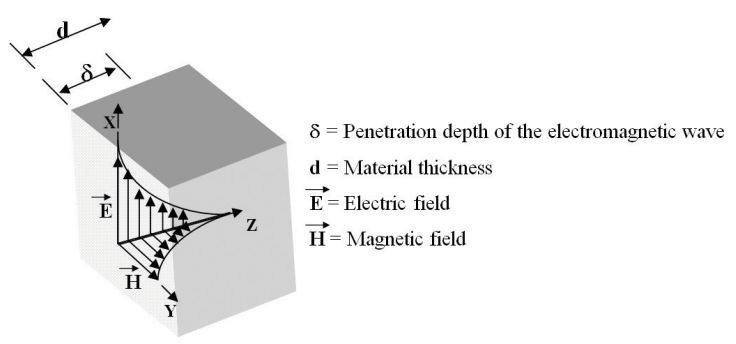

Figure 1. Decrease in the intensity of the electric and magnetic components of the electromagnetic field of a wave as it penetrates a material (After Balanis ${ }^{9}$ ).

\subsection{RAMs for stealth applications}

Radar absorbent materials are components of dual-use technology because they have applications in both the military and civilian industries. When used in military applications, RAMs are important elements in electronic warfare since their ability to attenuate return radar signals is of extreme importance. Stealth technology can also be used in civilian applications, especially in the area of electronic shielding and compatibility, for the reduction of electromagnetic interference and to control and minimize the levels of electromagnetic energy emitted by electric and electronic devices.

Most radars used for air-traffic control and air-defense systems operate in the microwave band (L, S, C, X and Ku bands); radars operating in the UHF, L, S and $\mathrm{C}$ bands are used to monitor the airspace over large distances. In this study, we prepared and evaluated materials that attenuate radar return signals in the $\mathrm{X}$-band frequency range (8 to $12 \mathrm{GHz}$ ). Radars operating in this frequency range are used to detect, identify and track targets with high resolution. Examples of X-band radars in operation are those used in land-based air defense systems and in missiles, which can of track targets as far as $90 \mathrm{~km}$ in clear skies (on rainy days, the radar range is reduced $)^{15-17}$. Therefore, in military applications, targets that are less visible in this frequency range have a tactical advantage.

\section{Material and Methods}

\subsection{Radar Absorbing Material preparation}

The RAM was prepared using a commercial laminated composite (TenCate Advanced Composites, Netherlands) and a CNT-based nanostructured coating. The laminated composite consisted of ten alternating layers of continuous GF and PPS; PPS is a high-performance thermoplastic matrix widely used in aeronautical applications. The total thickness of the laminated composite was $2.2 \mathrm{~mm}$ and its specific mass and $1.6 \mathrm{~g} / \mathrm{cm}^{3}$.

The nanostructured coating used to paint the laminated composite was produced by mixing multi-walled CNTs (Iljin Nanotech Co. Ltd., South Korea) with industrial grade polyurethane $[5.0 \%(\mathrm{w} / \mathrm{w})]$. The polyurethane and the multi-walled CNTs were mixed by mechanical agitation for $15 \mathrm{~min}$. A brush was used to apply the nanostructured coating to the surface of the laminated composite (Figure 2).

The nanostructured coating was cured at room temperature for $6 \mathrm{~h}$. The average thickness of the coating layer applied to the GF/PPS laminated composite was $0.15 \mathrm{~mm}$. The morphological characteristics of the material

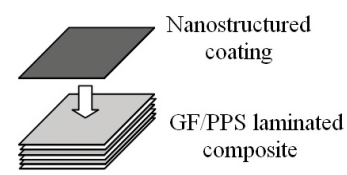

Figure 2. Painting of the GF/PPS laminated composite with the nanostructured coating. 
were examined with a scanning electron microscope (SEM, LEO 435 VPI), without special sample preparation.

\subsection{Electromagnetic characterization}

Reflectivity measurements of the GF/PPS laminated composite painted with the nanostructured coating, using the waveguide technique ${ }^{18}$ in the frequency range 8 to 12 $\mathrm{GHz},(\mathrm{X} \text {-band })^{19}$ are shown in Figure 3. The waveguide (Figure $3 \mathrm{~b}$ ) is coupled to a network vector analyzer equipped with an S-parameter test set $(45 \mathrm{MHz}-26 \mathrm{GHz}$, Model 8510C, Agilent Technologies, USA) (Figure 3a), which is connected to a synthesized frequency generator $(45 \mathrm{MHz}-$ 26,56 GHz, Hewlett Packard 8510A, USA). Dielectric and magnetic parameters (complex permittivity and complex permeability) are calculated from the measured values of the S-parameters (reflection and transmission coefficients, $S_{11}, S_{22}$ ) with a software provided by the manufacturer of the network vector analyzer. The reference material used to calibrate the measurements was an aluminum plate, assumed to be $100 \%$ reflective (Figure $3 \mathrm{c}$ ). Rectangular-shaped samples $(2.3 \times 1.1 \mathrm{~cm})$ that fitted exactly the cavity of the waveguide were prepared. Measurements of reflectivity and absorption were performed to obtain the S-parameters. In a later stage of this study, larger samples will be prepared so that the materials can be evaluated using the NRL arc technique and in anechoic chamber to simulate the response of the material to an actual radar and its behavior in open field conditions.

\section{Results and Discussion}

Figure 4 shows the GF/PPS laminated composite painted with the nanostructured coating. Because of the thinness of the coating $(0.15 \mathrm{~mm})$, the specific mass of the laminated composite was not changed significantly. SEM images of a sample of the nanostructured coating removed from the surface of the laminated composite after curing and of CNTs in the nanostructured coating are shown in Figure 5.

The general aspect of the CNTs before mixing with polyurethane is seen in Figure 5a. A homogeneous distribution of CNTs in the polyurethane resin was obtained by mechanical mixing. CNTs are visible in the other SEM images as bright structures or flecks. The surface of the nanostructured coating is shown in Figure $5 \mathrm{~b}$. In spite of the mechanical mixing, clustering of CNTs can be observed in Figure 5c. A region showing high concentration of CNTs is visible in Figure 5d. The SEM images and the general

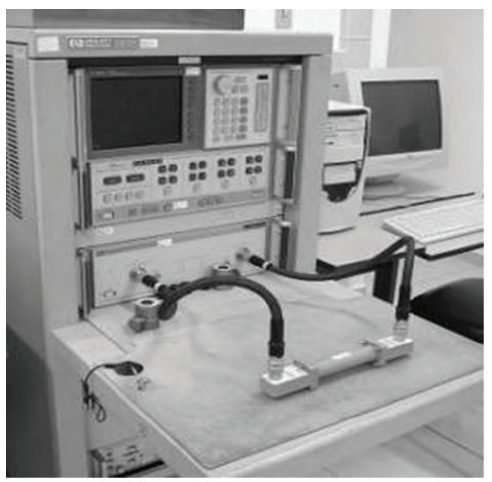

(a)

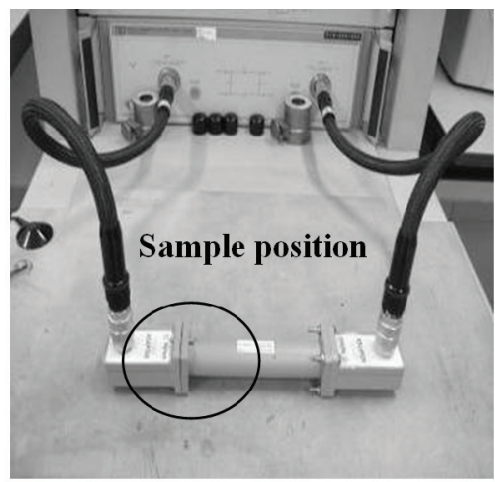

(b)

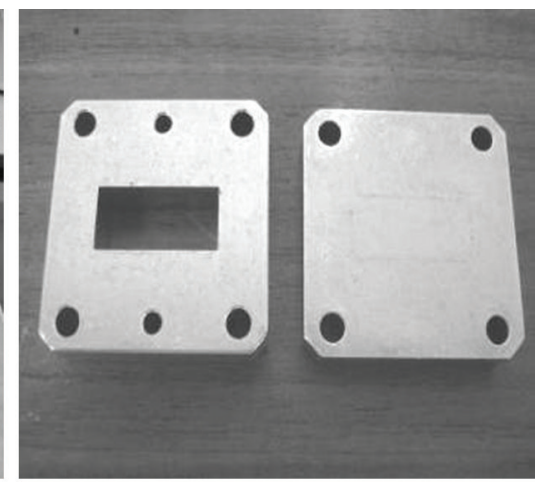

(c)

Figure 3. Equipment used for the electromagnetic characterization of RAMs: (a) network vector analyzer, (b) waveguide and sample position, (c) sample holder and aluminum plate.

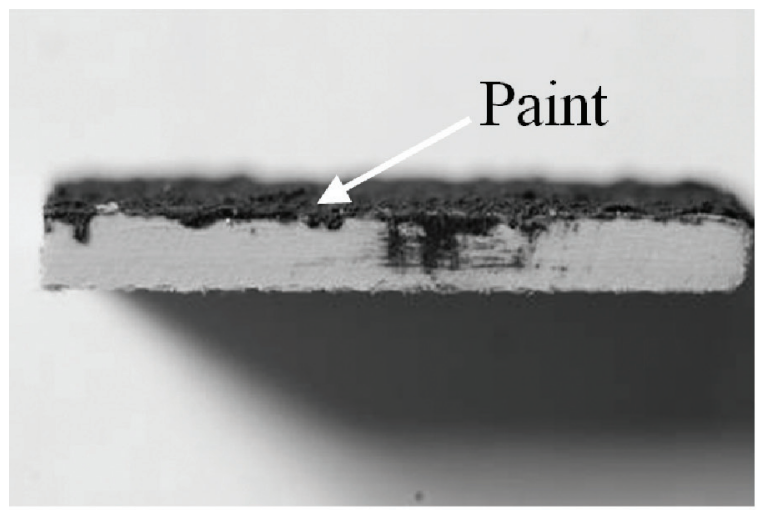

(a)

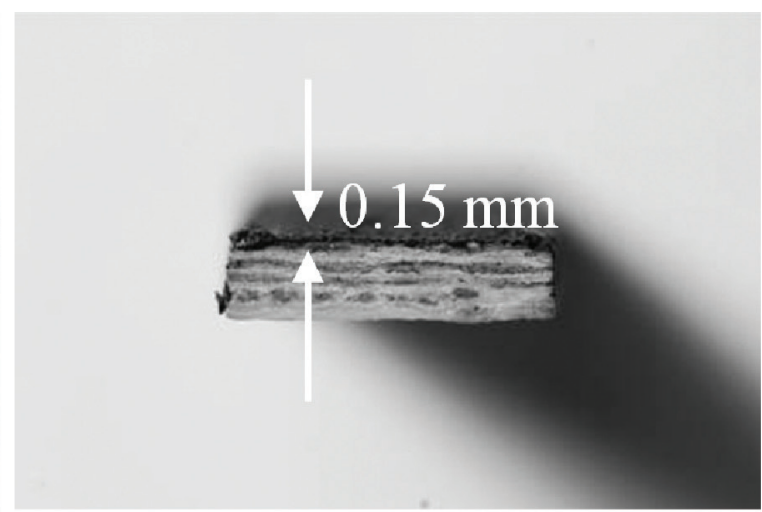

(b)

Figure 4. Morphological aspect of the GF/PPS laminated composite after painting with the nanostructured coating: (a) Cross section; (b) thickness of the nanostructured coating. 


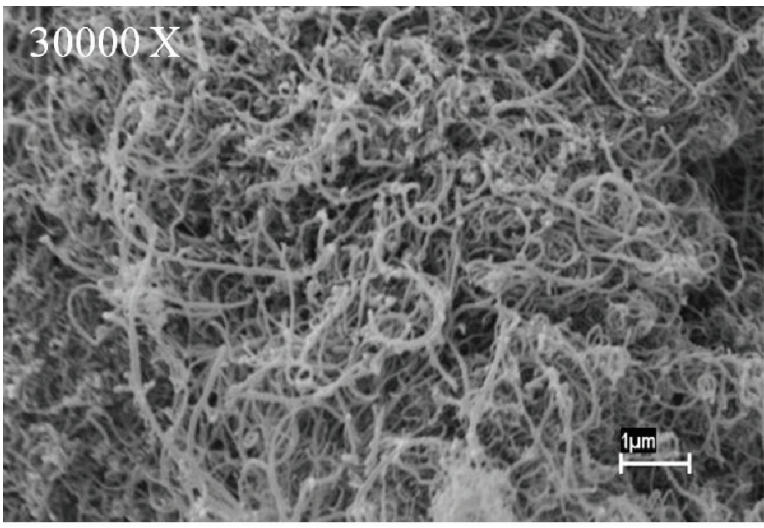

(a)

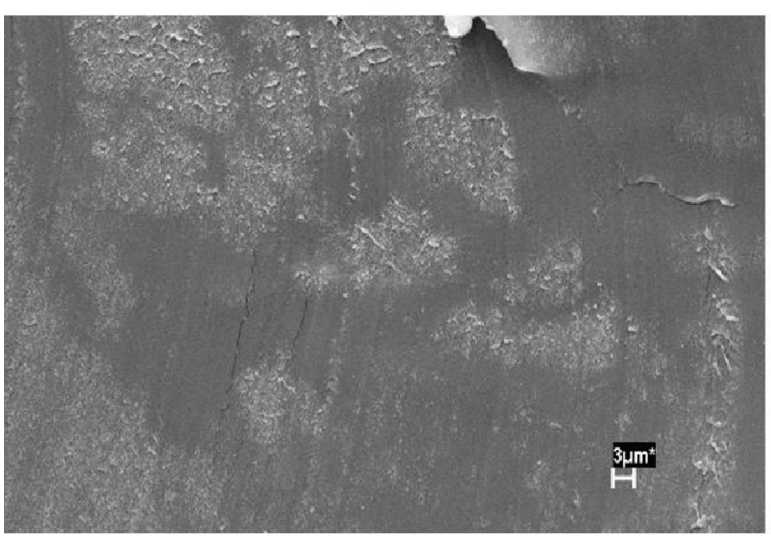

(c)

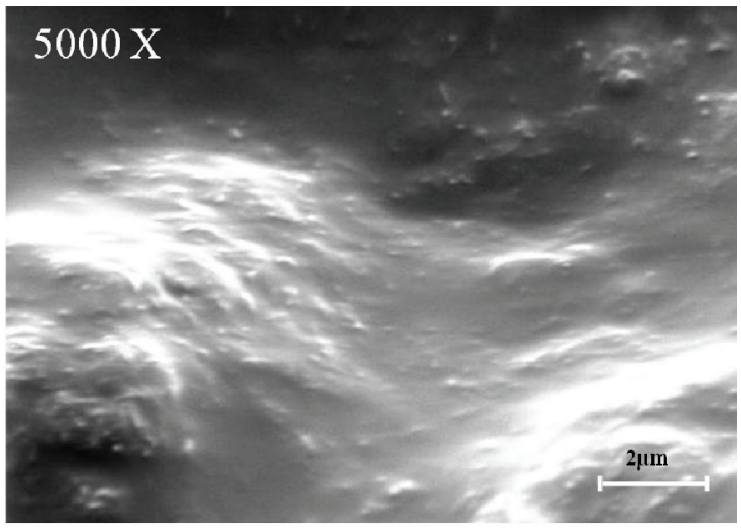

(b)

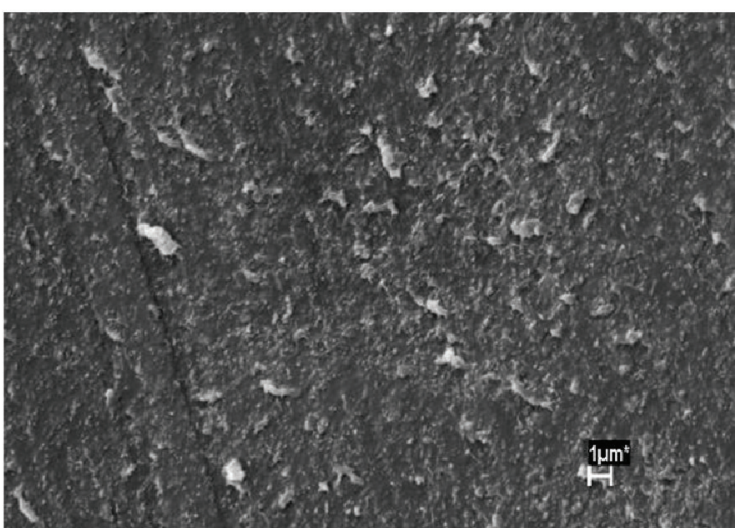

(d)

Figure 5. (a) CNTs used to produce the nanostructured coating, before mixing; (b) surface of the nanostructured coating; (c) region showing CNT clusters; and (d) region showing high concentration of CNTs.

composition of the material developed indicate that it has an amorphous structure.

Electromagnetic reflectivity measurements (frequency range, 8 to $12 \mathrm{GHz}$ ) and electromagnetic properties (complex permittivity and complex permeability) of the absorbing material prepared in this study are shown in Figures 6 and 7, respectively. Measurements of the reflectivity of the GF/PPS laminated composite without the nanostructured coating were made and revealed that the GF/ PPS laminated composite does not absorb electromagnetic radiation. When the GF/PPS laminated composite is placed over the aluminum plate, the system reflects $100 \%$ of the incident radiation.

Figure 6 shows that the GF/PPS laminated composite painted with the nanostructured coating in contact with the aluminum plate absorbed $87 \%$ (attenuation, $-9.0 \mathrm{~dB}$ ) of the energy of the incident radiation at $8 \mathrm{GHz}$ and $65 \%$ (attenuation, $-4.5 \mathrm{~dB}$ ) at $12 \mathrm{GHz}$. The absorption resonance frequency is located outside or at the lower limit of the X-band. Around $9.5 \mathrm{GHz}$ (frequency commonly used by acquisition and tracking radars), the absorption of energy of the incident radiation was approximately $80 \%$ (attenuation, $-7.0 \mathrm{~dB}$ ) and at $8.0 \mathrm{GHz}$ (the lower limit of the X-band) the absorption was $87 \%$ (attenuation, $-9.0 \mathrm{~dB}$ ). Absorbing materials can be grouped in two categories: resonant (or narrowband), and wideband absorbers. Resonant absorbers are more common and are usually made with a single layer of an absorbent material, while wideband absorbers are made by combining different materials in layered arrangements ${ }^{9}$. Usually, the resonance peak of absorbent materials is around $9.5 \mathrm{GHz}$, but the frequency of the resonance peak can be changed depending on the arrangement of layers in a multilayer absorbent material in such a way that the material absorbs over a wide frequency band.

Data in Figure 7 indicate that that the nanostructured coating behaves as a dielectric material. The real and imaginary values of the relative electric permittivity $\left(\varepsilon^{\prime} \approx 9.5\right.$ to $\left.8 ; \varepsilon^{\prime \prime} \approx 5.5\right)$ and relative magnetic permeability ( $\mu^{\prime} \approx 1, \mu^{\prime \prime} \approx 0$ ), which is similar to that of air, show that only dielectric losses are important. Dielectric losses within a material are related to the value of imaginary component of permittivity; large values of the imaginary permittivity result in large losses. A material in which dielectric losses are low can store electromagnetic energy but cannot dissipate it. On the other hand, a material in which dielectric losses are high does not store energy efficiently, a significant portion of the energy is converted into heat, i.e. the material absorbs energy and functions as a RAM. The complete description of the physical phenomena responsible for the variation of permittivity and permeability with frequency and the 


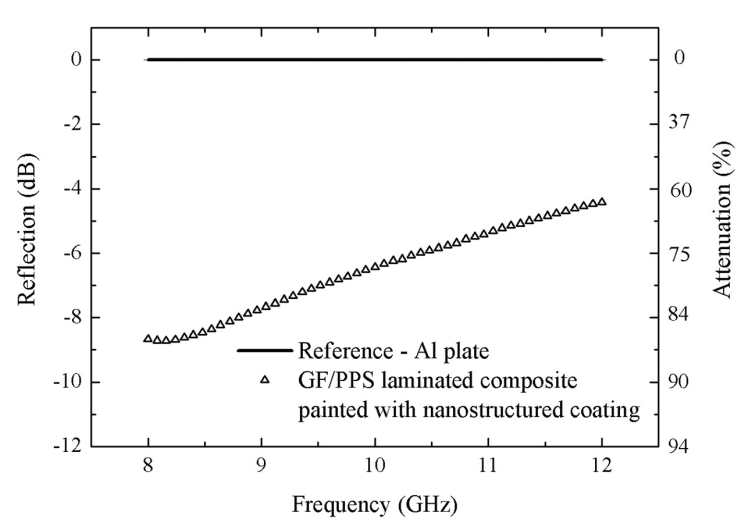

Figure 6. Reflectivity of the GF/PPS laminated composite painted with the nanostructured coating.

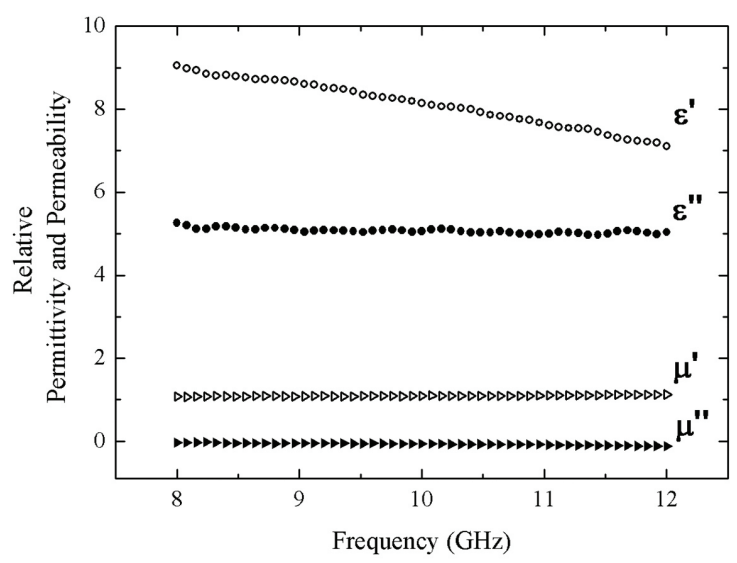

Figure 7. Complex electric permittivity (real part, $\varepsilon$ '; imaginary part, $\varepsilon ")$ and complex magnetic permeability (real part, $\mu$ '; imaginary part, $\mu$ ") of the GF/PPS laminated composite painted with the nanostructured coating.

absorption of energy by the RAM are complex topics whose explanation involves wave-matter interaction concepts that are beyond the scope of the present paper, the interested reader is referred elsewhere ${ }^{11}$.

As a comparison, 2-mm thick absorbing materials made with epoxy resin and nickel-zinc ferrite were evaluated in the frequency range 2 to $18 \mathrm{GHz}$, and the maximum attenuation (at the resonance peak, $7.5 \mathrm{GHz}$ ) was $-12 \mathrm{~dB}$. In the X-band, the attenuation ranged from $-11 \mathrm{~dB}(92 \%$ absorption of electromagnetic energy), at $8 \mathrm{GHz}$, to $-4 \mathrm{~dB}$ (60\% absorption of electromagnetic energy) at $12 \mathrm{GHz}^{[20]}$. It should be noted that ferrite-based absorbent materials have relatively high specific masses and, consequently, are not useful in aeronautical applications. The use of a dielectric component such as the conductive polymer polyaniline in place of the ferrite displaces the resonance peak into the X-band, the attenuation of the material becomes $-4 \mathrm{~dB}$ (60\% absorption) at the lower limit of the X-band frequency range, reaching a maximum value of $-12 \mathrm{~dB}$
(94\% absorption of electromagnetic energy) at $11.5 \mathrm{GHz}$. The material prepared and evaluated in this study not only is very thin, but it is also made using a formulation which does not change significantly the specific mass of the resulting composite material. Differently from other types of RAMs, the material developed in the present study is not applied to a surface but rather is used as a structural material. Its physical and chemical characteristics and good performance as an absorbent material suggest that it has many potential applications as a RAM.

\section{Conclusion}

In this study, we described the preparation of a RAM consisting of nanostructured coating (CNTs dispersed in a polyurethane matrix) applied to a $\mathrm{GF} /$ PPS laminated composite. Reflectivity measurements ( 8 to $12 \mathrm{GHz}$ ) of the GF/PPS laminated composite painted with the nanostructured coating showed that this material has the potential to be used as a microwave absorbing structural material. A relatively thin $(0.15 \mathrm{~mm})$ layer of the nanostructured coating with specific electromagnetic properties allowed the penetration of electromagnetic radiation into the material and absorption of up to approximately $90 \%$ of the energy of the incident electromagnetic wave. The absorbing centers (CNTs) used in the nanostructured coating changed the impedance of the material, transforming it in an effective RAM. The use of CNTs is a viable option for the production of RAM and RAS. Materials that are typically transparent to microwave and radar waves such as the GF/PPS laminated composite can become absorbers of electromagnetic energy when painted with CNTs-based coatings. Another important characteristic of the material prepared in this study is its low specific mass compared with that of conventional metallic-particle- or ferrite-based RAMs, which can increase the mass of a structure they are applied to by 4-5 $\mathrm{kg} / \mathrm{m}^{2}$ for absorption of $10 \mathrm{~dB}$ and higher. In the near future, we plan to extend this study to include laminate composites and paints with different thicknesses to determine the influence of the material thickness on its absorbing properties.

RAMs are produced with absorptions that suit their applications, from the shielding of electronic equipment to state-of-art coatings for high performance airplanes, so absorption can range from a few $\mathrm{dBz}$ to tens of $\mathrm{dBz}$. The combination of the mechanical strength of the composite and the absorbing properties of the nanocomposite paint resulted in a material with low specific mass that can simultaneously function as an absorber of electromagnetic radiation and as a structural element in many types of applications both in the civilian and military industries.

\section{Acknowledgment}

The authors wish to thank the Departamento de Ciência e Tecnologia Aeroespacial (DCTA) for the technical support and the Brazilian government funding agency CNPq (Project numbers: 559246/2008-0, 151803/2008-0 and 157949/2011-7) for financial. 


\section{References}

1. Thostenson ET and Chou T-W. Microwave processing: fundamentals and applications. Composites: Part A. 1999; 30:1055-1071. http://dx.doi.org/10.1016/S1359835X(99)00020-2

2. Folgueras LC and Rezende MC. Microwave absorbing nanocomposites composed with and without polyaniline by use as Radar Absorbing Structure. Materials Science Forum. 2013; 730-732:920-924. http://dx.doi.org/10.4028/ www.scientific.net/MSF.730-732.920

3. Folgueras LC, Alves MA and Rezende MC. Microwave absorbing paints and sheets based on carbonyl iron and polyaniline: measurement and simulation of their properties. Journal Aerospace Technology and Management. 2012; 2(1):6370. http://dx.doi.org/10.5028/jatm.2010.02016370

4. Lee SM. International Encyclopedia of Composites. New York: VCH Publishers; 1991.

5. Hull D. An introduction to composite materials. Cambridge University Press; 1981.

6. Chun AL. Carbon nanotubes: blacker than black. Nature Nanotechnology. 2008 jan. http://dx.doi.org/10.1038/ nnano.2008.29

7. Dalton AB, Collins S, Muñoz E, Razal JM, Ebron VH, Ferraris JP et al. Super-tough carbon-nanotube fibres. Nature. 2003; 423:703. http://dx.doi.org/10.1038/423703a

8. Folgueras LC, Alves MA and Rezende MC. Desempenho de materiais absorvedores de radiação multicamadas à base de tecidos de vidro e carbono. In: XIII Simpósio Latinoamericano de Polímeros y XI Congreso Iberoamericano de Polímeros (SLAP); 2012; Bogotá.

9. Balanis CA. Advanced Engineering Electromagnetics. 2nd ed. New York: John Wiley and Sons; 2012. p. 200.

10. Quéffélec P, Le Floc'h M and Gelin P. Broad-band characterization of magnetic and dielectric thin films using a microstrip line. IEEE Transactions on Instrumentation and Measurement. 1998; 47(4):956-963. http://dx.doi. org/10.1109/19.744649

11. Tsang L, Kong JA and Ding KH. Scattering of Electromagnetic Waves, Theories and Applications. New York: WileyInterscience; 2000.

12. Oh J-H, Oh K-S, Kim C-G and Hong C-S. Design of radar absorbing structures using glass/epoxy composite containing carbon black in $\mathrm{X}$-band frequency ranges. Composites: Part B. 2004; 35:49-56. http://dx.doi.org/10.1016/j. compositesb.2003.08.011

13. Rmili H, Miane JL, Zangar $\mathrm{H}$ and Olinga TE. Microwave conductivity measurements of high conductive polyaniline films. European Physical Journal Applied Physics. 2005; 29:6572. http://dx.doi.org/10.1051/epjap:2004208

14. Cao M, Qin R, Qiu C and Zhu J. Matching design and mismatching analysis towards radar absorbing coatings based on conducting plate. Materials and Design. 2003; 24:391-396. http://dx.doi.org/10.1016/S0261-3069(02)00119-X.

15. Knott EF, Shaeffer JF and Tuley MT. Radar Cross Section. 2nd ed. Norwood: Artech House; 1993.

16. Barton DK. Modern Radar System Analysis. London: Artech House; 1988.

17. Adamy D. EW 101: A First Course in Electronic Warfare. London: Artech House; 2001.

18. Nicolson AM and Ross G. Measurement of the intrinsic properties of materials by time domain techniques. IEEE Transactions on Instrumentation Measurement. 1970; 19(4):377-382. http:// dx.doi.org/10.1109/TIM.1970.4313932

19. Skolnik MI, editor. Radar Handbook. New York: McGraw-Hill Book Company; 2008. Chapter I.

20. Ting TH, Yu RP and Jau YN. Synthesis and microwave characteristics of polyaniline/NiZn ferrite composites in 2-40 GHz. Materials Chemistry and Physics. 2011; 126:364-368. http://dx.doi.org/10.1016/j.matchemphys.2010.11.011 